\title{
VIABILIDAD DE EXPLOTAR GAS SHALE EN ALGUNOS MUNICIPIOS DEL ESTADO DE TAMAULIPAS
}

\section{Cinthia Mariela Hernández Trejo}

\section{Daniel Romo Rico ${ }^{1}$}

\section{Resumen}

El gas natural se ha convertido en uno de los principales combustibles utilizados a nivel mundial. La revolución del gas shale ya se dio en Estados Unidos, ahora es conveniente utilizar las lecciones para propiciar en México su autosuficiencia energética y crecimiento económico. Este trabajo se enfoca en estudiar los retos para explotar dicho hidrocarburo por parte de las empresas petroleras en una parte de las regiones con alto potencial de gas de lutitas, como es la de Tampico-Misantla, definida inicialmente en la Ronda Uno licitación 4. Con base en el análisis deductivo de los principales factores que rodean esta actividad, se concluye sobre la necesidad de canalizar recursos de inversión y crear la infraestructura propia para su desarrollo y apoyo, así como considerar la problemática social enfrentada, en particular en materia de seguridad.

Palabras clave: Gas Shale, hidrocarburos, energía, desarrollo económico.

\section{Abstract}

Natural gas has become one of the main fuels used worldwide. The shale gas revolution has already occurred in the United States, it is now convenient to use the lessons to promote the Mexican energy self-sufficiency and economic growth. The research project focuses on studying the challenges to exploit such hydrocarbon by oil companies in one part of the regions with high potential for shale gas such as Tampico-Misantla early defined in Round One bidding 4. Based on the deductive

1 Profesores de la ESIA-Ticomán. Instituto Politécnico Nacional. 
analysis of the main factors surrounding this activity, we conclude that it is necessary to channel investment resources to create the infrastructure for development and support this activity, as well as to consider the social problems faced, especially in the security area.

Keywords: Gas Shale, hydrocarbons, energy, economy development.

\section{Introducción}

Desde la década de los noventa, el gas natural se ha convertido en uno de los principales combustibles utilizados a nivel mundial, debido a su bajo costo, ser menos contaminante que otros hidrocarburos en su combustión y a su relativa disponibilidad, amén de ser materia prima para la petroquímica. Se obtiene de yacimientos convencionales y no convencionales y se estima será el combustible que mayor penetración registrará como aporte de energía primaria. México produce insuficientes volúmenes de ese hidrocarburo para satisfacer su consumo interno, pero cuenta con un potencial considerable de reservas. Algunas de estas se encuentran distribuidas en el noreste del país, entre los que se destaca la cuenca Tampico-Misantla, en donde es posible acceder a su explotación empleando las tecnologías del fracking y la perforación horizontal.

En el marco de la instrumentación de la reforma energética, el gobierno mexicano anunció que como parte de la Ronda Uno, se licitarían campos no convencionales en la cuarta etapa que comprenden los municipios de Burgos, Casas, Cruillas, González, Jiménez, Llera, Padilla, San Carlos, San Nicolás, Victoria y Xicoténcatl. Aunque con los ajustes en el precio de los hidrocarburos, tal decisión se canceló, este trabajo se enfocó a contestar la pregunta ¿Existen las condiciones para que la iniciativa privada invierta en proyectos de explotación de shale gas en esa región? Para responderla, este estudio utiliza el método deductivo, de los temas asociados con el mercado del gas natural y de las condiciones para su explotación en esa región del país, en donde se incluye un análisis socioeconómico y político. Se concluye sobre la necesidad de atender distintos tópicos económicos y sociales para que las empresas petroleras particulares exploten el citado hidrocarburo en esa región. 


\section{Consideraciones Generales}

Al cierre de 2015, las reservas totales de gas natural en el mundo alcanzaron 186.9 billones de metros cúbicos (bpc), lo que significó 1.7 veces más que el nivel del año 1990. No obstante, se redujo el número de años de producción de 55.2 a 52.8. Una parte de esas reservas correspondió a campos no convencionales, los cuales alcanzaron su viabilidad económica gracias a los desarrollos tecnológicos en fracturación hidráulica y perforación horizontal ${ }^{2}$, particularmente en Estados Unidos. Se ha estimado que el total de reservas recuperables de gas en lutitas fue de 206.7 bmc en el mundo.

México es deficitario en la producción de gas natural, pues requirió importar 29.9 mil millones de metros cúbicos en 2015 para abastecer su consumo interno. Aunque cuenta con reservas potenciales, solo dispone del $0.2 \%$ del total mundial de las reservas probadas de ese combustible. El sector energético ha sido una de las fuentes de ingresos más importante para el Estado a través de la parte de renta petrolera. Con la reforma energética, esta será compartida con las empresas privadas, por lo que una mayor actividad de estas últimas podría ser de beneficio para las finanzas públicas, al tiempo que se pueden apuntalar los esfuerzos para diversificar las alternativas de posible abasto de gas natural.

Pemex, Exploración y Producción identificaron cinco provincias petroleras potencialmente productoras de gas y condensados de lutitas en el país: Sabinas, Burro-Picachos, Burgos MZ, Tampico-Misantla y Veracruz, en donde se estimaron recursos técnicamente recuperables de 60.2 mil millones de petróleo crudo equivalente (De la Vega, 2013). Una de las provincias petroleras con mayores reservas de aceite fue Tampico-Misantla con 30.7 miles de millones de barriles de crudo equivalente.

2 La perforación horizontal tiene como objetivo, atravesar y llegar a los yacimientos en diversos tipos de formaciones como las naturalmente fracturadas, de baja permeabilidad, estratificadas y lenticulares, entre otras. Mientras que la fractura hidráulica, incrementa la permeabilidad de la roca permitiendo la liberación del gas, esto se logra a través de reacciones químicas y el empleo de presión con fluidos 
Como parte de la Reforma Energética se definieron las áreas a licitar para la exploración de campos no convencionales de aceite y gas natural, asociados principalmente a la madurez del Queroseno y el Carbón Orgánico Total en la Roca de las Formaciones Pimienta del Jurásico Superior Tithoniano y Agua Nueva del Cretácico Superior Turoniano de la Provincia Petrolera Tampico-Misantla.

Pemex, Exploración y Producción han realizado algunos trabajos geológicos y geoquímicos en áreas prospectivas de aceite, gas húmedo y seco en esa zona, pero no ha desarrollado tecnología para la extracción de gas shale ${ }^{3}$, como otras empresas de servicio, por ejemplo, Schlumberger Canadá Ltd., Baker Hughes, Halliburton Co. o las productivas como las Oil Major (ExxonMobil, BP, Shell, Chevron y Total) o las empresas norteamericanas líderes en el tema como EOG Resources, Devon Energy y Chesapeake Energy, entre otras, algunas de las cuales son potenciales participantes en el mercado mexicano.

\section{Cuadro 1. Principales países con reservas recuperables de no convencionales}

\begin{tabular}{|l|c|}
\hline \multicolumn{2}{|c|}{ Aceite de Lutitas } \\
\hline País & $\begin{array}{c}\text { Miles de } \\
\text { millones } \\
\text { de barriles }\end{array}$ \\
\hline Rusia & 75 \\
\hline $\begin{array}{l}\text { Estados } \\
\text { Unidos }\end{array}$ & 58 \\
\hline China & 32 \\
\hline Argentina & 27 \\
\hline
\end{tabular}

\begin{tabular}{|l|c|}
\hline \multicolumn{2}{|c|}{ Gas en Lutitas } \\
\hline País & $\begin{array}{c}\text { Miles de } \\
\text { millones } \\
\text { de barriles }\end{array}$ \\
\hline China & 31.6 \\
\hline Argentina & 22.7 \\
\hline Argelia & 20.0 \\
\hline $\begin{array}{l}\text { Estados } \\
\text { Unidos }\end{array}$ & 18.8 \\
\hline
\end{tabular}

3 En el caso de México, solo se cuenta con 6 patentes: CARBO CERAMICS INC (Empresa Estadounidense); MX2006PA011762A. Enfocada al Fluido-Apuntalante; MINERAC O CURIMBABALTDA (Empresa Brasileña); MX2005PA011664AEnfocada al Fluido-Apuntalante; Schlumberger Technology bv; MX2005PA000443A. Enfocada a la medición de la fractura durante el proceso; MX2003PA003374A. Enfocada al método de empleo del agente apuntalante; MX2003PA007291A. Enfocada al Fluido apuntalante; RHONE POULENC CHIMIE (Empresa Francesa) y MX2003PA007291A. Enfocada al Fluido apuntalante. Fracturación de pozos para extracción de gas, COMINSA. 


\begin{tabular}{|l|c|}
\hline Libia & 26 \\
\hline Australia & 18 \\
\hline Venezuela & 13 \\
\hline México & 13 \\
\hline Pakistán & 9 \\
\hline Canadá & 9 \\
\hline Resto & 65 \\
\hline Total & 345 \\
\hline
\end{tabular}

\begin{tabular}{|l|c|}
\hline Canadá & 16.2 \\
\hline México & 15.4 \\
\hline Australia & 12.4 \\
\hline Sudáfrica & 11.0 \\
\hline Rusia & 8.1 \\
\hline Brasil & 6.9 \\
\hline Resto & 43.5 \\
\hline Total & 206.7 \\
\hline
\end{tabular}

Fuente: Comisión Nacional de Hidrocarburos (abril 2015).

Las modificaciones constitucionales a los artículos 25, 26 y 27 de diciembre de 2013 y sus leyes secundarias publicadas en agosto del año 2014, permitieron a las empresas privadas intervenir en la exploración, extracción y producción de hidrocarburos. Ello mediante las nuevas modalidades en los contratos de utilidad compartida, de producción compartida y licencias con el Estado Mexicano y/o con PEMEX, a cambio del pago de contraprestaciones como regalías, cuotas contractuales en la fase exploratoria, bono a la firma y una porción de las utilidades mediante el cobro del Impuesto Sobre la Renta (ISR). La explotación de campos de gas de lutitas o shale gas está comprendida dentro de tal iniciativa. En el nuevo marco regulatorio, también se abrieron espacios a las operaciones en Midstream para las compañías particulares, que es una actividad fundamental en la cadena de valor de la explotación del gas natural. 
Figura 1. Áreas para la exploración de recursos no convencionales en la Provincia petrolera Tampico-Misantla (Ronda 4)

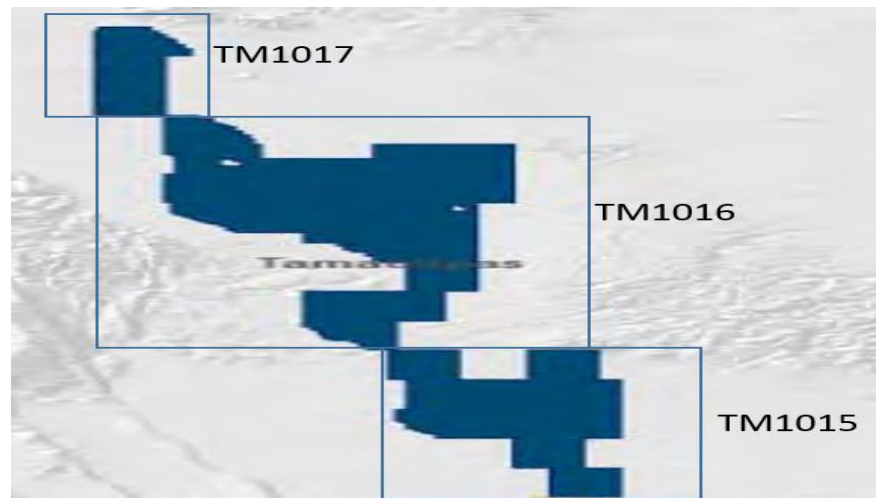

Fuente: Plan Quinquenal de Licitaciones para la Exploración y Extracción de Hidrocarburos 2015-2019

La Secretaria de Energía (SENER) conduce la política energética del país para garantizar el suministro competitivo y ambientalmente sustentable de energéticos, y del gas natural en particular. Esta función es realizada en conjunción con otras instituciones gubernamentales, como la Comisión Nacional de Hidrocarburos, que se encarga de asesorar y operar las actividades de Upstream. El órgano regulador de los términos y condiciones para prestación del servicio de distribución de gas natural, así como las tarifas correspondientes es la CRE (Comisión Reguladora de Energía). De igual forma la CRE emite la metodología correspondiente para fijar el precio de venta de primera mano del gas natural y su distribución al usuario final. La Agencia Nacional de Seguridad Industrial y de Protección al Medio Ambiente de Sector de Hidrocarburos (ASEA), regula y supervisa la seguridad industrial, operativa y de protección del medio ambiente, las instalaciones y actividades del sector hidrocarburos, incluyendo el desmantelamiento y abandono de instalaciones, así como el control de residuos. Finalmente, el Centro Nacional de Control del Gas Natural (CENAGAS) -organismo descentralizado de la Administración Pública Federal y sectorizado a la SENER- que actúa como Gestor del Sistema de Transporte y Almacenamiento Nacional Integra- 
do de Gas Natural (SISTRANGAS) y como transportista de gas natural, operando y manteniendo ductos propios.

\section{Cuadro 2. Recursos Prospectivos, 2014}

\begin{tabular}{|l|c|c|c|}
\hline \multicolumn{4}{|c|}{ Recursos prospectivos documentados } \\
\hline Provincia & $\begin{array}{c}\text { Aceite } \\
\text { mmmb }\end{array}$ & $\begin{array}{c}\text { Gas } \\
\text { mmmmpc }\end{array}$ & $\begin{array}{c}\text { PCE } \\
\text { mmmbpce }\end{array}$ \\
\hline Tampico- Misantla & 30.7 & 20.7 & 34.8 \\
\hline Burgos MZ & 0.0 & 53.8 & 10.8 \\
\hline Burro-Picachos & 0.6 & 18.0 & 4.2 \\
\hline Sabinas & 0.0 & 49.0 & 9.8 \\
\hline Veracruz & 0.6 & 0.0 & 0.6 \\
\hline Total Shale & 31.9 & 141.5 & 60.2 \\
\hline
\end{tabular}

Fuente: Comisión Nacional de Hidrocarburos (abril 2015).

PEMEX dejo de ser paraestatal para operar como empresa productiva del Estado, quitándole el monopolio que mantuvo desde 1938 en la industria petrolera nacional. Como contraprestación, se le asignaron en la Ronda Cero un conjunto de campos entre los que destacaron 5,225 mmbpce de reservas de gas de lutitas, que correspondieron alrededor del 9\% del total de reservas nacional de ese tipo de campos, volumen inferior al solicitado por la petrolera. Los campos asignados pueden ser explotados por la petrolera estatal o en conjunto con otras compañías, previa solicitud de su migración a nuevos contratos, lo que ofrece un área de oportunidad para que acceda a la tecnología. 


\section{Gráfica 1. Los 10 Principales Titulares de la tecnología para Fracturación de Pozos}

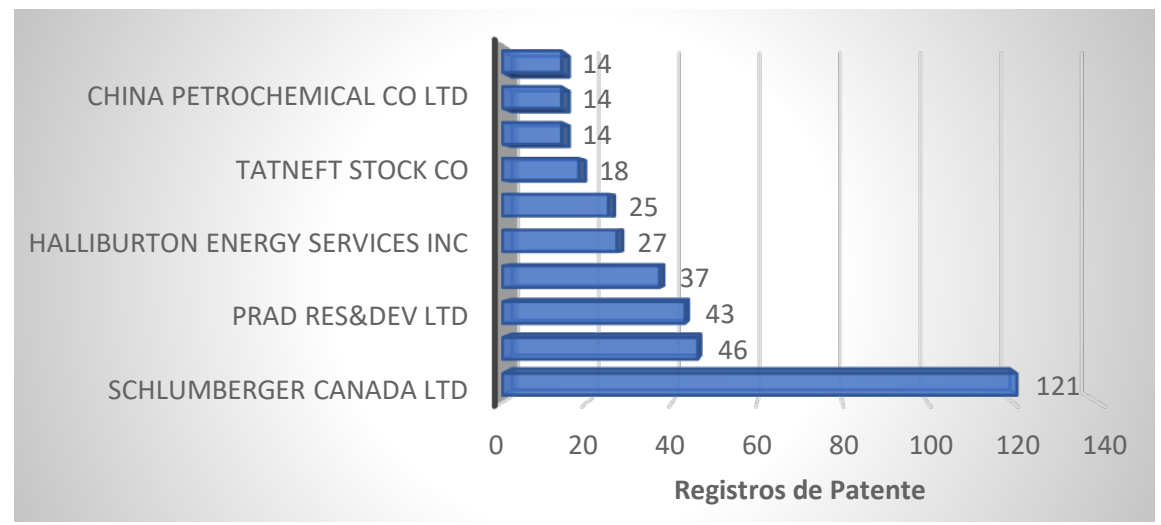

Fuente: Elaboración propia con base en la Corporación Mexicana de Investigación en Materiales, S.A. de C.V.

\section{La viabilidad de producción de gas de lutita en áreas de Tampico-Misantla}

La Provincia Petrolera Tampico-Misantla (PPTM), se ubica en la margen centro-oriental de México y comprende desde el extremo sur del Estado de Tamaulipas hasta la parte central de Veracruz, las porciones orientales de los estados de San Luis Potosí, Hidalgo, Norte de Puebla y occidente del Golfo de México hasta la isobata de 200 metros (m). Limita al norte con las provincias Burgos y Alto de Tamaulipas, al sur con el Cinturón Plegado de la Sierra Madre Oriental y la Faja Volcánica Transmexicana, al occidente con la Plataforma Valles-San Luis Potosí y el Cinturón Plegado de la Sierra Madre Oriental y al oriente con el Cinturón Extensional Quetzalcóatl. El Carbono Orgánico Total en la formación es de $0.5-8 \%$, por lo que el potencial generador se encuentra entre regular y muy rico. La profundad está entre los 1,500 metros y los 4,100 metros (Raitano Lucy, 2017).

Tamaulipas, donde se encuentran la mayor cantidad de recursos de Gas Shale de la Provincia Petrolera Tampico-Misantla, comprende los 
municipios de: Burgos, Casas, Crujillas, González, Jiménez, Llera, Padilla, San Carlos, San Nicolás, Victoria, Xicoténcatl, que son los lugares de análisis del presente estudio.

\section{Condiciones para la explotación del gas de lutitas}

PEMEX inició en 2010 actividades de explotación de gas shale con una serie de pozos que le han permitido asimilar paulatinamente el conocimiento para el desarrollo de esas actividades, algunas de las cuales han ocurrido en la Cuenta Tampico-Misantla ${ }^{4}$.

En general, los costos de perforación de gas de lutita están en función de una serie de factores, entre los que destacan localización geográfica, profundidad, presión e infraestructura disponibles para realizar las operaciones como para transportar el producto, así como del costo de las materias primas empleadas en esas actividades. No obstante, también deben considerarse costos derivados de factores externos, por la complejidad y riesgos afrontados. El gas de esquisto se extrae por la alta presión para fracturar la roca. Una cantidad significativa de esta agua vuelve a la superficie como el flujo de retorno dentro de los primeros días o semanas después de la inyección y se acompaña de grandes cantidades de metano.

El precio del gas natural en el país está referenciado al mercado estadunidense, con tendencia a niveles bajos en los últimos años, lo que ha limitado la rentabilidad de las operaciones de explotación del gas natural y resalta la importancia del empleo de tecnología para abatir los costos de producción.

Las técnicas de perforación y fractura hidráulica se han estado perfeccionando, modificando las perspectivas del sector energético, sin embargo, existen retos que se han enfrentado en el desarrollo de sus ac-

4 Las actividades desarrolladas por PEMEX en materia de campos no convencionales iniciaron con la perforación del pozo Emergente-1, Montañés-1, el Nómada-1, Percutor-1, Habano-1, Arbolero-1, Anhélido-1, Batial-1, Nerit-1, Mosquete-1, Serbal-1, Céfiro-1 y Tagram-1. Se tienen dos pozos prospectos (Durián y Arbolero-1) en el área de estudio. 
tividades, algunas de las cuales se derivan del negocio petrolero y otras derivadas de tales operaciones, las cuales se analizan a continuación.

\section{Gráfica 2. Sismos por año en Tampico-Misantla}

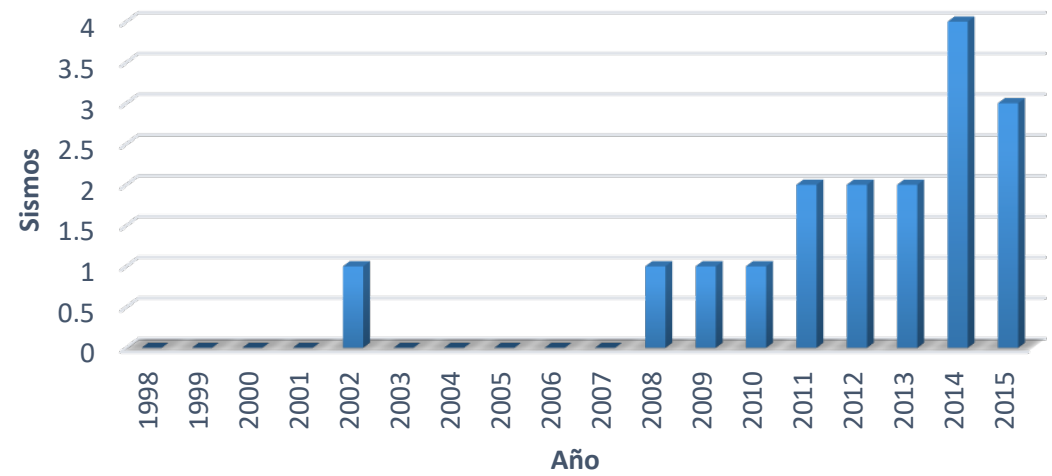

Fuente: Servicio Sismológico Nacional, elaborado por CartoCrítica, 2015

\section{Sísmica}

México se encuentra dividido en cuatro zonas sísmicas. El Estado de Tamaulipas se encuentra dentro de la zona A, que no tenía registros históricos de sismos, pero debido al fracking se ha incrementado la actividad sísmica en las provincias geológicas (Gráfica 2). Los sismos registrados en Tampico-Misantla no superan una magnitud considerable que ocasione daños notables, pues van desde 2.6 a 4.2 , que a menudo se sienten, pero causan daños menores.

\section{Contaminación}

Después de la terminación, algunas emisiones fugitivas continúan durante su vida útil. Un pozo típico tiene de 55 a 150 conexiones con equipos tales como calentadores, medidores, deshidratadores, compresores y aparatos de recuperación de vapores. Muchos de ellos con fugas y válvulas de alivio de presión y están diseñados para ventilar a propósito el gas. Las emisiones de las bombas neumáticas y deshidratadores son también una parte fuente de fuga. 


\section{Gráfica 3. Magnitud de los sismos por año en Tampico-Misantla}

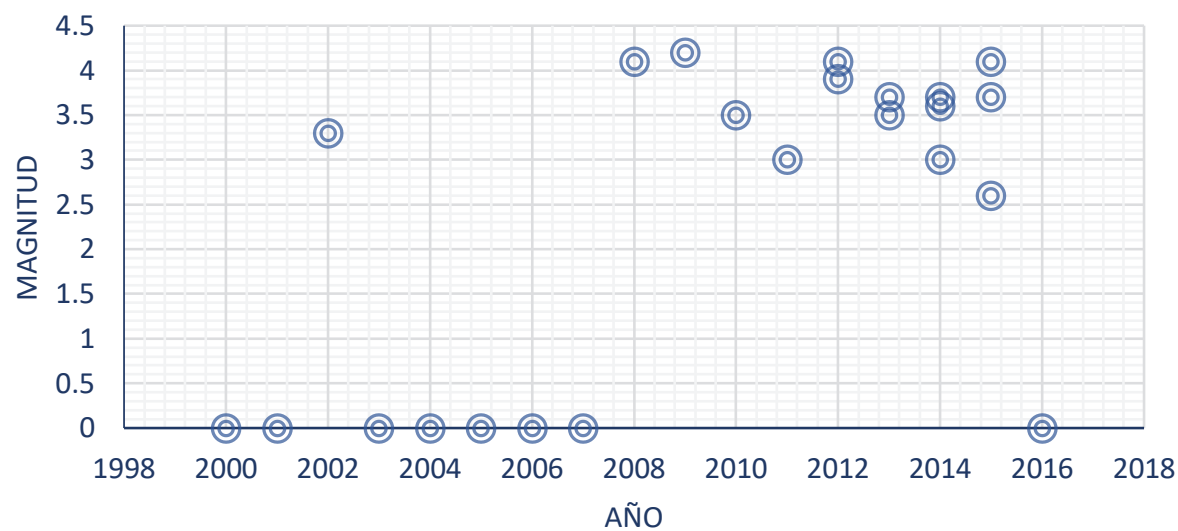

Fuente: CartoCrítica, 2015 (http://www.cartocritica.org.mx)

El gas natural en algunas ocasiones es de una calidad suficiente para ser utilizado sin más procesamiento, y en algunos casos contiene cantidades de hidrocarburos pesados e impurezas, tales como gases de azufre que deben ser retirados a través de procesamiento y por lo que existe una fuga de metano durante este procesamiento. Otras emisiones fugitivas se producen durante el transporte, almacenamiento y distribución del gas.

La huella de gases de efecto invernadero del gas shale es significativamente mayor que la del obtenido en campos convencionales, ya que las pérdidas estimadas, durante el ciclo de vida del gas shale son de 3.6 a $7.9 \%$ (Cuadro 3). La huella del gas shale es al menos 20\% mayor al gas convencional.

\section{Cuadro 3. Emisiones fugitivas de metano asociadas con el desarrollo de gas natural a partir de yacimientos convencionales} y no convencionales, en pozos de los Estados Unidos

\begin{tabular}{|l|c|c|}
\hline \multicolumn{1}{|c|}{ Concepto } & Gas Convencional & Gas Shale \\
\hline $\begin{array}{l}\text { Emisiones durante la } \\
\text { terminación del pozo }\end{array}$ & $0.01 \%$ & $1.9 \%$ \\
\hline $\begin{array}{l}\text { Ventilación y fugas de } \\
\text { rutina en equipos en el } \\
\text { sitio del pozo }\end{array}$ & $0.3 \mathrm{a} 1.9 \%$ & $0.3 \mathrm{a} 1.9 \%$ \\
\hline
\end{tabular}




\begin{tabular}{|l|c|c|}
\hline $\begin{array}{l}\text { Emisiones durante la } \\
\text { descarga de líquido }\end{array}$ & 0 a $0.26 \%$ & 0 a $0.26 \%$ \\
\hline $\begin{array}{l}\text { Emisiones durante el } \\
\text { procesamiento de gas }\end{array}$ & 0 a $0.19 \%$ & 0 a $0.19 \%$ \\
\hline $\begin{array}{l}\text { Emisiones durante el } \\
\text { transporte, almacena- } \\
\text { miento y distribución }\end{array}$ & 1.4 a $3.6 \%$ & 1.4 a $3.6 \%$ \\
\hline Emisiones Totales & 1.7 a $6.0 \%$ & 3.6 a $7.9 \%$ \\
\hline
\end{tabular}

Fuente: Elaboración propia con base en datos de Methane and the greenhouse-gas footprint of natural gas from shale formations, 2011

\section{Agua}

El agua es uno de los temas más preocupantes y polémicos en la extracción del gas shale, ya que durante el fracturamiento hidráulico, su cantidad empleada oscila entre los 9,800 litros hasta más de 36 millones de litros por pozo (USGS, 2015). El principal uso del agua en el estado de Tamaulipas es el agrícola con el $54 \%$, seguido con el $34 \%$ es la generación de energía. El agua residual en el Estado es de alrededor de $706,557,145 \mathrm{~m}^{3}$ al año.

El volumen de agua empleada en Tamaulipas fue de 6.4 millones de $\mathrm{m}^{3}$. Sus principales usos fueron para la agricultura y para la generación energía eléctrica durante el año 2013 (Cuadro 4). Los municipios en estudio dependen hidrológicamente y en distinta medida de los ríos Soto la Marina, Támesi y San Fernando principalmente, pero no se considera que tengan excedentes de agua ${ }^{5}$, por lo que una alternativa para la extracción del gas shale podría ser transportándola desde otros lugares o bien empleando agua de mar, lo que elevaría los costos, por su tratamiento, transporte y almacenamiento.

5 Si la cantidad de agua empleada por pozo es de un máximo de 36 millones de metros cúbicos $\left(\mathrm{m}^{3}\right)$ y la consumida en el Estado de Tamaulipas de 6,363.8 millones de $\mathrm{m}^{3}$, entonces el agua utilizada para la fractura hidráulica cubriría para 177 pozos por año. 


\section{Cuadro 4. Uso del agua en diversas actividades en Tamaulipas $\mathrm{m}^{3} / \mathbf{a} \tilde{n} o$}

\begin{tabular}{|l|c|}
\hline \multicolumn{1}{|c|}{ Uso } & Volumen Total \\
\hline Agrícola & $54.13 \%$ \\
\hline Agroindustrial & $0.00 \%$ \\
\hline Doméstico & $0.02 \%$ \\
\hline Acuacultura & $0.69 \%$ \\
\hline Servicios & $0.08 \%$ \\
\hline Industrial & $2.66 \%$ \\
\hline Pecuario & $0.10 \%$ \\
\hline Público Urbano & $5.24 \%$ \\
\hline Múltiples & $2.80 \%$ \\
\hline Generación de Energía & $34.28 \%$ \\
\hline Otros & $0.00 \%$ \\
\hline Total & $100.00 \%$ \\
\hline
\end{tabular}

Fuente: CONAGUA, noviembre 2015

Existe el riesgo potencial de la contaminación de los acuíferos cercanos, cuando se presentan fallas en la estructura del pozo inyector (cementación y revestimiento) y la posibilidad de contaminación con las sustancias químicas adicionadas al agua de fracturamiento o por el hidrocarburo extraído ${ }^{6}$. Los insumos tóxicos utilizados en el proceso de fracturamiento y los lodos pueden afectar la productividad de los suelos, y al lixiviarse, llegar a contaminar los acuíferos subyacentes.

Una vez culminado el procedimiento de fractura, el fluido utilizado regresa a la superficie, en proporciones que varían, dependiendo del pozo de entre un $15 \%$ y un $80 \%$ del total inyectado. Este fluido de retorno puede ser reutilizado para la fractura hidráulica, poniendo como referencia el valor máximo de agua por pozo $\left(36,000 \mathrm{~m}^{3}\right)$, el volumen de reflujo de agua suponiendo un $80 \%$ es de $28,800 \mathrm{~m}^{3}$.

6 Se han identificado más de 750 tipos de químicos en el fluido de perforación, algunos de los cuales algunos se consideran cancerígenos, otros pueden afectar al sistema endócrino, causar daños en el sistema nervioso, o provocar alergias (SEMARNART, 2015). 
Todo esto hace considerar que la cantidad de agua disponible para los diversos usos de los seres humanos, podría disminuir por las actividades del fracking. Por lo anterior, conviene utilizar tecnología que permita el uso de aguas residuales o tratadas para el proceso de fracking, así como la reutilización del agua para el mismo proceso e incluir tecnología más avanzada que permitan disminuir su uso.

En cada proceso de fractura se generan numerosos volúmenes de desechos líquidos que pueden contener, además de agua, arena y los químicos utilizados. Existe el riesgo de contaminación de aguas subterráneas durante el proceso, principalmente por fugas de gas metano y por los químicos utilizados ${ }^{7}$. Estos fluidos de desecho no son recuperados en su totalidad y son fuente potencial de daños al medio ambiente, y de problemas sociales. También existe la posibilidad de contaminación del agua, por el derrame de las balsas que almacenan el fluido de retorno, pues es agua procesada con químicos y queda contaminada. En caso de hacer un mal cálculo de este fluido de retorno o por causa de lluvias, esta se desborda contaminando aguas y terrenos. Entonces, el principal problema durante el proceso de fracking está en desaparecer estas aguas contaminadas de una manera que no afecte al medio ambiente, lo ideal sería reutilizarla.

El reto en la explotación de gas shale es la utilización de químicos con un mínimo de toxicidad y peligrosidad, para disminuir los riesgos a la salud y al medio ambiente que provienen del empleo de ese tipo de aditivos $^{8}$. En Estados Unidos se han logrado avances para abatir tal problemática, sin embargo, resultan costos adicionales que las compañías

7 El flujo de retorno generado por la fractura hidráulica contiene diversas sustancias toxicas procedentes del subsuelo como materiales pesados (arsénico, plomo, cromo, mercurio), radioactivas de origen natural (uranio, radio, radón), bencenos (habitualmente en forma de benceno, tolueno, etilbenceno y xileno) y grandes concentraciones de sales.

8 El principal reto tecnológico radica en producir alternativas de manejo seguro de sustancias químicas, remediación de sitios contaminados y sobre todo conservar la disponibilidad de agua limpia para el uso humano. 
podrían enfrentar, por lo que son relevantes las acciones de vigilancia y seguimiento que realicen las autoridades gubernamentales.

Existe otro tipo de afectaciones a la biodiversidad, como el desmonte de áreas, construcción de caminos, polvo, ruido y el tráfico de vehí$\operatorname{culos}^{9}$ que pueden afectar a las especies vegetales y animales. Estudios sugieren que la fracturación de pozos es la fuente de actividades sísmicas presentadas en las zonas cercanas a las plataformas de perforación, donde anteriormente no se habían registrado movimientos, por lo cual el estudio de éstos debe ser elaborado previo, durante y post apertura de un pozo de extracción de gas ${ }^{10}$.

Debido a que la industria del gas no puede manejar las grandes cantidades de aguas residuales originadas por la fractura hidráulica, es frecuente que emplee pozos de inyección (también llamados pozos letrina) para deshacerse del agua contaminada. Estos pozos pueden desestabilizar fallas geológicas y provocar sismos.

\section{Entorno socioeconómico y político en el área de estudio para el desarrollo de la explotación del gas shale}

En 2014, el Producto Interno Bruto (PIB) de Tamaulipas se elevó a más de 495 mil millones de pesos, algo así como el 3\% del PIB nacional. La principal actividad fue comercio y servicios, que contribuyeron con el $50 \%$ del PIB estata ${ }^{11}$. La actividad industrial aportó un $47 \%$ del PIB Estatal, de las cuales un $25 \%$ estuvo relacionada con el sector energé-

9 Se han detectado afectaciones a la infraestructura carretera y habitacional por el tránsito de camiones de carga en por carreteras y caminos vecinales.

10 Un estudio (Matthew, 2015) demuestra que la producción de salmuera combinado con la inyección de aguas residuales produce cambios en el subsuelo, y tomando en consideración los datos históricos de la sismicidad de la región, sugiere que los cambios de tensión subterráneos están asociados a los procesos de la fractura hidráulica, representando la causa más probable de los terremotos en el área de Azle, Texas.

11 El comercio contribuyó con el 15\%, los servicios inmobiliarios (12.8\%) y transportes, correos y almacenamiento $(10.2 \%)$. 
tico e incluyeron la extracción de hidrocarburos, refinación, la generación, transmisión y distribución de energía eléctrica y la fabricación de productos derivados en la industria petroquímica (22\% del PIB de Tamaulipas). El 3\% correspondió a actividades primarias. Hacia finales de noviembre de 2016 contaba con un poco más de 128 mil unidades económicas.

\section{Gráfica 4. Población Municipios de Tamaulipas en área de Estudio, 2015}

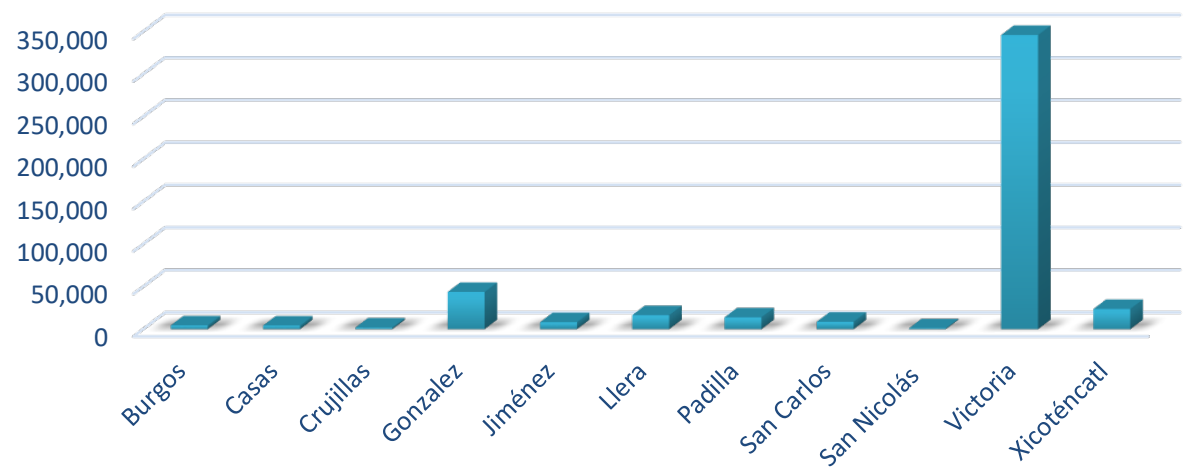

Fuente: Elaboración propia con base en datos del INEGI

El régimen de propiedad de la tierra es mixto, pero impera la pequeña propiedad, a excepción de Llera y Victoria, que registran una mayor participación relativa de propietarios ejidales (Cuadro 5). Esta organización de la posición de la tierra permite abrir espacios a las operaciones de explotación de shale gas en esos municipios para las empresas, en mejores condiciones, que donde prevalece el régimen de propiedad ejidal.

La disponibilidad de mano de obra es crítica para el desarrollo de la explotación de los campos no convencionales, tanto la empleada directamente que requiere un nivel de calificación técnico, como la que se utiliza de manera indirecta. La posibilidad de explotar el gas shale en el área de estudio, puede estar soportada por una relativa disponibilidad de mano de obra, alguna de la cual está formada en las actividades asociadas a la industria petrolera. Al año 2010, el Estado de Tamaulipas 
contaba con una población de 3,268,554 personas $^{12}$, de las cuales el $88 \%$ habitaba en áreas urbanas, por arriba del promedio nacional. El total de la población en los municipios en estudio representó el 14\% del total (Grafica 4), y de esta, la mayor parte se concentró en la capital del Estado. Cerca del 38\% de la población se ubicaba en situación de pobreza y una tercera parte en pobreza moderada.

\section{Cuadro 5. Régimen de Propiedad de la Tierra}

\begin{tabular}{|l|c|c|c|c|}
\hline Municipio & $\begin{array}{c}\text { Extensión } \\
\text { territorial } \\
\text { (Hectáreas) }\end{array}$ & $\begin{array}{c}\% \text { del territorio } \\
\text { de Tamaulipas }\end{array}$ & $\begin{array}{c}\% \text { propiedad } \\
\text { ejidal }\end{array}$ & $\begin{array}{c}\% \text { pequeña } \\
\text { propiedad }\end{array}$ \\
\hline Burgos & 224,162 & $2.81 \%$ & $7 \%$ & $93 \%$ \\
\hline Casas & 364,373 & $5.12 \%$ & $14 \%$ & $86 \%$ \\
\hline Cruillas & 161,840 & $2.03 \%$ & $21 \%$ & $79 \%$ \\
\hline González & 339910. & $4.26 \%$ & $19 \%$ & $81 \%$ \\
\hline Jiménez & 192,740 & $2.41 \%$ & $11 \%$ & $89 \%$ \\
\hline Llera & 228,353 & $2.86 \%$ & $41 \%$ & $57 \%$ \\
\hline Padilla & 135,126 & $1.69 \%$ & $25 \%$ & $75 \%$ \\
\hline San Carlos & 269,204 & $3.37 \%$ & $11 \%$ & $89 \%$ \\
\hline San Nicolás & 72,280 & $0.91 \%$ & $7 \%$ & $93 \%$ \\
\hline Victoria & 153,829 & $2.04 \%$ & $35 \%$ & $65 \%$ \\
\hline Xicoténcatl & 87,761 & $1.58 \%$ & $36 \%$ & $64 \%$ \\
\hline
\end{tabular}

Fuente: Elaboración Propia con base en datos de Plan Municipal de Ordenamiento Territorial y Desarrollo Urbano

El promedio de escolaridad entre 2013-2014 fue de 9.4 con un índice de analfabetismo de $3.3 \%$ en el Estado de Tamaulipas. Alrededor del $16 \%$ de la población registraba rezago educativo en 2014 . Alrededor de 1,500 egresados al año como técnicos superiores, de ésos el 73\% de los pertenecían al área de Ingeniería, Manufactura y Construcción.

12 La población total del Estado creció en un 5\% de 2010 a 2015 a 3,441,698 personas, en 2010. INEGI, Principales Resultados del Censo de Población y Vivienda 2010, Tamaulipas. 
La Población Económicamente Activa de Tamaulipas llego a 1,616 mil personas, con una tasa de ocupación del $94.2 \%$, durante el cuarto trimestre del 2013. Del total de la Población Económicamente Activa Ocupada 18,214 personas laboraban en el Sector Energético (Cuadro 6).

\section{Cuadro 6. Población Económicamente Activa dedicada al Sector Energético en el Estado de Tamaulipas (número)}

\begin{tabular}{|l|c|}
\hline \multicolumn{1}{|c|}{ Actividad } & Población \\
\hline $\begin{array}{l}\text { Fabricación de equipo de generación y distribución } \\
\text { de energía eléctrica }\end{array}$ & 20,126 \\
\hline $\begin{array}{l}\text { Construcción de obras para el suministro de agua, } \\
\text { petróleo, gas, energía eléctrica y telecomunicaciones }\end{array}$ & 19,103 \\
\hline Industria del plástico y del hule & 11,714 \\
\hline Extracción de petróleo y gas & 10,717 \\
\hline $\begin{array}{l}\text { Fabricación de productos derivados del petróleo y } \\
\text { del carbón }\end{array}$ & 6,563 \\
\hline Industria química & 6,234 \\
\hline $\begin{array}{l}\text { Generación, transmisión y distribución de energía } \\
\text { eléctrica }\end{array}$ & 3,897 \\
\hline $\begin{array}{l}\text { Suministro de agua y de gas por ductos al consumi- } \\
\text { dor final }\end{array}$ & 353 \\
\hline Transporte de gas por ductos & 113 \\
\hline Total & 78,820 \\
\hline
\end{tabular}

Fuente: Agenda Energética de Tamaulipas 2014

Los egresados de licenciatura fueron de alrededor de 15 mil estudiantes anualmente, correspondiendo el $30.5 \%$ a las citadas áreas ${ }^{13}$. Sin embargo, además de su nivel académico, se presentan retos de su experiencia y habilidades en la explotación de los campos de shale gas. Existe una capacidad relativa para ofrecer de recursos humanos en el área petro-

13 En Tamaulipas existen 96 universidades con 4 programas de Doctorado, 11 de Maestría y 51 programas de Licenciatura vinculados con el Sector Energético. De esas 35 se encuentran en Cd. Victoria, la capital del Estado, una en Gonzales y una en Burgos (CIEES). 
lera, sobresaliendo el área de geociencias del Instituto Tecnológico de Ciudad Madero y siete escuelas de Ingeniería Petrolera ${ }^{14}$.

El Estado contaba con 320 unidades de Salud, de las cuales en los municipios con recursos de Gas Shale se encuentran 47. Si bien existe infraestructura básica para atender población, el nivel de los servicios es insuficiente y con retos de eficiencia, particularmente en los servicios públicos (Instituto Nacional de Salud Pública, 2013). Por lo anterior, sería necesario aumentar la infraestructura en materia de salud, sobre todo en hospitales de especialidades, a fin de atender a la potencial población.

Para el desarrollo de las actividades de explotación del gas de lutitas, se requiere de vías de comunicación que permitan transportar el agua, los químicos y materiales empleados. Se considera que el Estado de Tamaulipas tiene insuficiente infraestructura para atender el desarrollo de esas actividades, por lo que sería necesario ampliarla. La longitud total de la Red Nacional de Carreteras según superficie de rodamiento en 2013 en el Estado fue de 14,008 kilómetros, de las cuales 5,319 estaban pavimentadas, 8,503 kilómetros estaba revestida y 186 kilómetros de terracería. La longitud total de las vías férreas fue de 936.7 kilómetros, pero 683,9 kilómetros son troncales y ramales, 167,9 kilómetros son vías secundarias y 84,9 kilómetros pertenece a vías particulares.

Al cierre del 2015, el Estado contaba con 5 aeropuertos Internacionales ${ }^{15}$ y con cuatro Puertos Marítimos, de los cuales tres eran comerciales y uno es pesquero. Cerca de los municipios en estudio, se encuentra el aeropuerto de Cd. Victoria, y no a gran distancia el de Tampico donde pasa el sistema troncal de carreteras en el Estado y existen otras carreteras regionales y caminos de terracería, que permitirían el tránsito a pozos petroleros potenciales.

14 Instituto de Ciencias y Estudios Superiores de Tamaulipas; Instituto de Estudios Superiores de Tamaulipas; Universidad Autónoma de Tamaulipas; Universidad del Norte de Tamaulipas; Universidad Tamaulipeca, Universidad del Noreste y el Centro Educacional y Desarrollo en Informática Personal.

15 Los aeropuertos existentes en el estado de Tamaulipas son el de Tampico, Cd. Victoria, Matamoros, Reynosa y Nuevo Laredo) 
Además, de impulsarse las actividades petroleras en los campos no convencionales, otras actividades económicas podrían estimularse, como la industria hotelera, comercial, servicios y de la construcción, con los consecuentes beneficios para la población de la región.

\section{Figura 2. Infraestructura Energética de Tamaulipas}

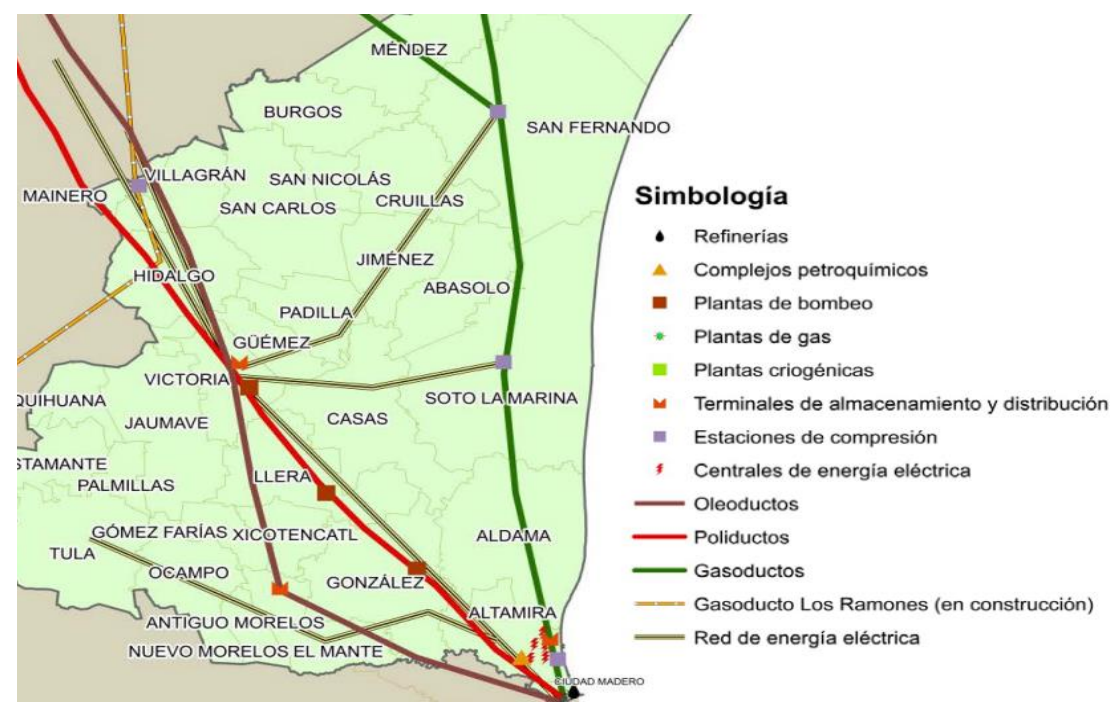

Fuente: Agenda Energética de Tamaulipas 2014.

En Tamaulipas pasa un Gasoducto por el Este y está en proceso de construcción el Gasoducto los Ramones, que pasara $52 \mathrm{~km}$ por el Norte y Oeste de Tamaulipas, aunque no en los municipios de estudio. Esto representa una ventaja por la posibilidad de conectarse a un gasoducto eje del país, y aprovechar el acceso a menor costo de transporte ${ }^{16}$.

El Estado tiene una sede de la Alianza Mexicana contra el Fracking y "No Fracking Tamaulipas", quienes han enumerado los perjuicios que

16 El transporte de gas natural en el Bakken Shale tiene un costo por barril de $\$ 10$ a $\$ 13$ dolares en tren, mientras que el transporte por gasoductos puede llegar a diminuir su costo hasta $\$ 5$ dolares por barril. (EIA, 2016 b). 
se originan de la explotación del gas de lutitas, y son un grupo de la Sociedad Civil, que estaría en contra del desarrollo de la citada actividad.

\section{Cuadro 7. Características de Bakken y Tampico Misantla}

\begin{tabular}{|l|c|c|}
\hline \multicolumn{1}{|c|}{ Características } & Tampico-Misantla & Bakken \\
\hline TOC & $0.5-8 \%$ & $11.33 \%$ \\
\hline Kerogeno & II y III & I, II y III \\
\hline Profundidad (metros) & $1500-4100$ & 1371.6 a 2286 \\
\hline Madurez Térmica & $455^{\circ} \mathrm{C}$ & $425^{\circ} \mathrm{C}$ \\
\hline
\end{tabular}

Fuente: Elaboración propia con base en datos de PEMEX (2012) y Hui Jin (2014).

Uno de los grandes retos que enfrenta el Estado es la violencia, entre los de mayor grado del país, a causa del crimen organizado. Las incidencias delictivas en 2015 en los municipios de interés fueron relevantes. De los 3,060 delitos patrimoniales el 10\% fueron hechos en el área de estudio, $17 \%$ de los Delitos Sexuales, 10\% de los homicidios, 5\% de las lesiones, 9\% otros delitos, 24\% de los Secuestros, $12 \%$ de los robos comunes y $12 \%$ de los robos en carreteras (Cuadro 8). Durante 2016, la percepción de inseguridad se encontraba por arriba del promedio nacional 83.9 (vs 73.3) (CONCANACO SERVYTUR, 2016). Ello, junto con los niveles de corrupción, han sido factores que han agudizado el descontento social.

El partido que gobernaba de manera mayoritaria (Partido Revolucionario Institucional) perdió las elecciones por la gubernatura y en varios municipios del área de estudio ${ }^{17}$. En el marco de este proceso, no se considera que el cambio de poder pueda ser un factor de influencia negativa para realizar inversiones en la industria petrolera, sin embargo, el

17 En las elecciones del 2013 predominaba el PRI, empero el partido político que predomino en las elecciones municipales en 2016, el Partido Acción Nacional, ganó 6 municipios de los 11 del área de estudio, la coalición del Partido Revolucionario Institucional (PRI), Partido Verde Ecologista (PVEM) y el Partido Nueva Alianza (PANAL) 4 municipios, y 1 por un candidato independiente. pero en estas elecciones del 2016 le quito varios municipios el PAN, al igual que la gubernatura del Estado. 
tema de la inseguridad debe ser contemplado como parte de los costos, en caso de llevarse a cabo proyectos de perforación de pozos en los campos de lutitas.

Pemex Exploración y Producción considera que Tampico-Misantla tiene similitud con la formación que Bakken Shale, que es una formación rocosa del Devónico Tardío, a principios de la edad del Mississippi, constituida por capas de lutitas y areniscas y una superficie de 6,522 millas cuadradas que se encuentra a profundidades de 4,500 a 7,500 pies de profundidad con una media de 6,000 pies y un espesor medio de 22 pies, porosidad del $8 \%{ }^{18}$. Ha enfrentado problemas técnicos por el declive acelerado de la producción, lo que se agudizó porque el precio del petróleo disminuyó desde finales del 2014, y la rentabilidad de las operaciones se vio afectada.

Entre los retos que se enfrentan en esos campos, que pudieran enfrentarse en el caso mexicano son: problemas para mantener la producción y la necesidad de perforar una considerable cantidad de pozos anualmente, y, por tanto, la canalización de inversiones; incremento de las emisiones de metano, provocando un daño al aire; altos niveles de contaminantes y sustancias toxicas en aguas por derrames de salmuera; sismos inducidos de escasa escala, que no afectan las actividades económicas y humanas; $y$ encontrar procesos eficientes que permitan la reutilización del agua residual para la re-fractura hidráulica, aminorando la inyección, almacenaje y transporte de aguas residuales ${ }^{19}$.

Sin embargo, se considera que es una referencia técnica para la explotación de gas shale en el área de estudio, y en donde se espera que la producción de petróleo alcance 1.3 millones de barriles diarios en 2019

18 El Bakken Shale el tipo de Kerogeno va desde I que es potencialmente petrolífero, pero también puede producir gas y Kerogeno II capaz de generar aceite y gas principalmente, pero es un poco más profundo existe Kerogeno III capaz de generar gas.

19 El declive en la producción de Bakken Shale se ha originado por el bajo número de plataformas trabajando. 
superando a Eagle Ford y convertirse en el más grande productor de petróleo shale en Estados Unidos (EIA, 2016).

\section{Cuadro 8. Índices Delictivos, 2015}

\begin{tabular}{|l|c|c|c|c|c|c|}
\hline & \multicolumn{2}{|c|}{ Victoria } & \multicolumn{2}{c|}{$\begin{array}{c}\text { Resto munici- } \\
\text { pios en estudio }\end{array}$} & \multicolumn{2}{c|}{$\begin{array}{c}\text { Edo. } \\
\text { Tamaulipas }\end{array}$} \\
\hline $\begin{array}{l}\text { Delitos } \\
\text { patrimoniales }\end{array}$ & 234 & $7 \%$ & 59 & $7 \%$ & 3,060 & $7 \%$ \\
\hline $\begin{array}{l}\text { Delitos } \\
\text { sexuales }\end{array}$ & 79 & $2 \%$ & 11 & $1 \%$ & 531 & $1 \%$ \\
\hline Homicidios & 67 & $2 \%$ & 65 & $8 \%$ & 1,279 & $3 \%$ \\
\hline Lesiones & 111 & $3 \%$ & 93 & $11 \%$ & 4,253 & $10 \%$ \\
\hline Otros delitos & 819 & $25 \%$ & 349 & $40 \%$ & 13,027 & $32 \%$ \\
\hline $\begin{array}{l}\text { Priv. De la } \\
\text { libertad }\end{array}$ & 42 & $1 \%$ & 14 & $2 \%$ & 230 & $1 \%$ \\
\hline Robo común & 1,912 & $59 \%$ & 272 & $31 \%$ & 18,628 & $45 \%$ \\
\hline $\begin{array}{l}\text { Robo en } \\
\text { carreteras }\end{array}$ & 4 & $0 \%$ & 2 & $0 \%$ & 52 & $0 \%$ \\
\hline Total & 3,268 & $100 \%$ & 865 & $100 \%$ & 41,060 & $100 \%$ \\
\hline
\end{tabular}

Resto municipios en estudio: Burgos, Casas, Crujillas, González, Jiménez, Llera, Padilla, San Carlos, San Nicolás y Xicoténcatl

Fuente: Elaboración Propia con base en datos de Incidencia Delictiva 2015, Secretariado Ejecutivo

\section{Conclusión}

La tendencia mundial sobre el consumo de gas natural como fuente de energía va en aumento en los últimos años y sigue creciendo, porque es un medio de abastecimiento de combustible de bajo costo, amigable con el medio ambiente y de disponibilidad relativa abundante. El desarrollo del fracking y la perforación horizontal, permitieron dar viabilidad a los yacimientos no convencionales para poder cubrir la demanda mundial de este hidrocarburo, particularmente en los campos de Estados Unidos en donde se ha apoyado el desarrollo de esa actividad. 
A pesar de que México cuenta con significativas reservas, adquiere del exterior una parte proporción creciente de su consumo interno de gas natural. Con la reforma energética de 2013, se tiene la oportunidad de acceder a su explotación por parte de empresas privadas, tanto en campos convencionales como no convencionales. El reto es ofrecerles las condiciones de rentabilidad para operar. La explotación de Gas Shale en la cuenca de Tampico-Misantla se presenta como una alternativa para robustecer la producción de Gas Natural en México, pues es una formación con espesores ricos en contenido orgánico y madurez térmica de la edad Jurásico-Cretácico, con potencial de recursos de gas/oil shale.

El presente trabajo se centró, primordialmente, en estudiar la viabilidad de explotar los campos de gas shale existentes en 11 municipios del Estado de Tamaulipas: Burgos, Casas, Cruillas, González, Jiménez, Llera, Padilla, San Carlos, San Nicolás, Victoria, Xicoténcatl.

Con independencia del nivel de precios de los hidrocarburos, y del gas natural en particular, que se ubican en niveles cercanos a los costos de producción en varios campos no convencionales, se identificaron un conjunto de retos técnicos, sociales, económicos, ambientales y geológicos por superar para acceder a la explotación del gas shale en esa región:

- No se cuenta con la tecnología adecuada para su extracción en el país. Está en proceso de asimilación por parte de PEMEX, pero existe potencialidad de acceso de compañías privadas internacionales, principalmente del vecino país del norte.

- El uso intensivo del agua durante el proceso de fracking puede constituirse un obstáculo. No existen excedentes de ese líquido en la zona, siendo el principal destino el consumo humano que, de no atenderse, podría generar descontento social. Ello se subsanaría transportando agua de mar a las zonas de actividad petrolera, pero con costos adicionales.

- Es necesario construir adicional infraestructura para transportar y procesar el gas natural y los insumos requeridos para la perforación de los pozos, pues no se dispone de los ductos necesarios para ampliar las actividades de explotación de gas shale, 
así como de la suficiente infraestructura de caminos y medios de comunicación.

- Aunque no se estiman problemas sociales para acceder a los terrenos en donde se localicen los pozos, pues la mayoría de ellas son pequeña propiedad, existe la posibilidad de que se genere distorsión en sus precios, lo que encarecería los costos de producción.

- Un reto mayúsculo es la inseguridad, particularmente en la capital del Estado, lugar natural para la ubicación de las oficinas de las empresas. De hecho, Tamaulipas es uno de los Estados más peligrosos del país.

- Se cuenta con infraestructura básica en materia de salud, pero al igual que en el resto del país está concentrada en las grandes ciudades, por lo que se requerirá de robustecer el gasto de capital para ampliar las inversiones, sobre todo para la atención de especialidades.

- En materia de recursos humanos, existen carreras ligadas al desarrollo de la explotación de campos no convencionales, sin embargo, se consideran insuficientes y con la necesidad de adicional experiencia y conocimiento en una actividad poco desarrollada en el país, como es la explotación de gas shale.

- El desarrollo de pozos de explotación de gas shale, podría generar una derrama de actividades por la necesidad de adicionales caminos, infraestructura hotelera, de salud, empleos, pago de impuestos y contribuciones, entre otros (Jianu, 2015), pero sobre todo la posibilidad de ampliar la oferta de gas natural en el país.

- Es conveniente contemplar los efectos sobre el medio ambiente a lo largo de la cadena de producción de los campos de lutitas. Posible contaminación de suelo y agua o suelos por derrames se pueden traducir en descontento social y en argumentos de apoyo para los grupos de la sociedad en el Estado en contra de tales actividades, como la Alianza Mexicana contra el fracking y la "No Fracking Tamaulipas". 
Finalmente, Bakken Shale es un área que contiene hidrocarburos no convencionales, que presenta similitud con la cuenca Tampico-Misantla, según PEMEX. Esto ofrece contar con una referencia para la perforación de pozos, que es conveniente atender, sobre todo por la variabilidad del precio del gas natural y la necesidad de obtener tasas atractivas de rentabilidad que permitan la sustentabilidad de las operaciones.

\section{Cuadro 9. Recursos Hídricos en el área de Estudio de Tamaulipas}

\begin{tabular}{|c|c|c|}
\hline Municipio & Población & Recurso hídrico \\
\hline Burgos & 4,428 & $\begin{array}{l}\text { Río Conchos y arroyos de caudal permanente como el } \\
\text { Burgos y Mojarras }\end{array}$ \\
\hline Casas & 4,179 & $\begin{array}{l}\text { Principalmente del arroyo y de grandes números de afluen- } \\
\text { tes. La Presa Vicente Guerrero ocupa una mínima porción } \\
\text { de la superficie municipal. }\end{array}$ \\
\hline Cruillas & 1,937 & $\begin{array}{l}\text { Varios arroyos que enriquecen el caudal del río Conchos, } \\
\text { contándose entre los más importantes El Mimbres y Las } \\
\text { Chorreadas. }\end{array}$ \\
\hline González & 43,757 & $\begin{array}{l}\text { Río Guayalejo y Río Tamesí, también se cuenta con la pre- } \\
\text { sa Ramiro Caballero, San Lorenzo y Venustiano Carranza. }\end{array}$ \\
\hline Jiménez & 8,165 & $\begin{array}{l}\text { Principalmente el Arroyo Flechadores, y otros arroyos: } \\
\text { Carretas, El Cazador Azufrosa, El Carrizo y la Serna. }\end{array}$ \\
\hline Llera & 16,555 & $\begin{array}{l}\text { El río Guayalejo, de caudal permanente; es enriquecido } \\
\text { por los escurrimientos de los arroyos de Santa Clara, Las } \\
\text { Adjuntas y Lucio Blanco. El municipio cuenta con la presa } \\
\text { El Módulo. }\end{array}$ \\
\hline Padilla & 13,927 & $\begin{array}{l}\text { Recursos hidrológicos muy elevados por ubicarse el } \\
\text { territorio en la cuenca del río Purificación, llevando su } \\
\text { caudal a la presa Vicente Guerrero. Los ríos y arroyos más } \\
\text { importantes que desembocan en la presa son: San Carlos, } \\
\text { Pilón, Corona, San Marcos, Santa Rosa, Arroyo Sarnoso y } \\
\text { el Grande. }\end{array}$ \\
\hline San Carlos & 8,723 & $\begin{array}{l}\text { Río San Carlos, el cual capta los arroyos del Lobo, La } \\
\text { Azufrosa, el Dulce, el Salado y Noche Buena. }\end{array}$ \\
\hline $\begin{array}{l}\text { San } \\
\text { Nicolás }\end{array}$ & 1,038 & $\begin{array}{l}\text { Río de San Nicolás que tras un buen recorrido se vierte al } \\
\text { río Conchos o San Fernando. }\end{array}$ \\
\hline
\end{tabular}




\begin{tabular}{|l|l|l|}
\hline Victoria & 346,029 & $\begin{array}{l}\text { Numerosos escurrimientos provenientes de la Sierra Madre } \\
\text { Oriental. En la porción media se localiza el río Santa Ana o } \\
\text { río Caballeros y los arroyos San Felipe, La Presa, Aquiles } \\
\text { Serdán y Santa María. El área urbana de la cabecera muni- } \\
\text { cipal es atravesada por el río San Marcos y hacia el sur los } \\
\text { arroyos Juan Capitán, Ojo Caliente y El Sauz. }\end{array}$ \\
\hline Xicoténcatl & 23,739 & $\begin{array}{l}\text { El río Guayalejo cruza el municipio de noreste a suroes- } \\
\text { te, además cuenta con la presa Xicoténcatl y de la presa } \\
\text { Emilio Portes Gil, mismas que son alimentadas por el río } \\
\text { Guayalejo. }\end{array}$ \\
\hline
\end{tabular}

Fuente: Elaboración Propia con base en datos de Hidrografía, Gobierno del Estado de Tamaulipas.

\section{Bibliografía}

ANUIES, Asociación Nacional de Universidades e Instituciones de Educación Superior. En: www.anuies.mx [Consultado 12 de abril 2016].

CIEES, Comités Interinstitucionales para la Evaluación de la Educación Superior. En: http://www.ciees.edu.mx/ [Consultado 15 de octubre 2016].

COMINSA, Fracturación de Pozos para Extracción de Gas, expediente 387. En: http://www.comimsa.com.mx/cit/data/GasShale/ ESTUDIO\%20fracturacion\%20de\%20pozos-v2.pdf [Consultado 25 de diciembre 2016].

De la Vega, Angel, "El Gas de Lutitas (Shale Gas) en México. Recursos, explotación, usos”, Revista Economíaunam, vol. 12, núm. 34, 2013, pp. 79-105.

DOF, "Ley de Ingresos sobre Hidrocarburos", Diario Oficial de la Federación. En: http://www.diputados.gob.mx/LeyesBiblio/pdf/ LIH_060117.pdf [Consultado 20de diciembre 2015].

EIA, Energy Information Administration, "Annual Energy Outlook 2015", USA, 2016. 
EIA, Energy Information Administration, "Trends in U.S. Oil and Natural Gas Upstream Costs". En: https:/www.eia.gov/analysis/ studies/drilling/ [Consultado 9 de octubre 2016].

Estrada, Javier A. Desarrollo del gas lutita (shale gas) y su impacto en el mercado energético de México: reflexiones para Centroamérica, Naciones Unidas, México, 2013, p. 118.

Horner, R.M., Harto, C.B., Jackson, R.B., Lowry, E.R., Brandt, A.R., Yeskoo, T.W., Murphy, D.J., Clark, C.E., (2016), "Water Use and Management in the Bakken Shale Oil Play in North Dakota", Environ Sci Technol, 2016, pp. 3275-3282.

INAFED, Instituto Nacional para el Federalismo y el Desarrollo Municipal, "Enciclopedia de los Municipios y Delegaciones de México. 2010". En: http://www.inafed.gob.mx/work/enciclopedia/ [Consultado 5 de noviembre 2015].

INEGI, Instituto Nacional de Estadística y Geografía, "Principales Resultados del Censo de Población y Vivienda 2010. Tamaulipas". En: http://www.beta.inegi.org.mx/proyectos/ccpv/2010/ [Consultado 22 de octubre 2015].

, Instituto Nacional de Estadística y Geografía, Perspectiva Estadística de Tamaulipas, México, 2012 http://internet. contenidos.inegi.org. $\mathrm{mx} / \mathrm{contenidos/productos/prod \_ serv/}$ contenidos/espanol/bvinegi/productos/integracion/estd_perspect/ dic_2013/tamps/702825055585.pdf, [25/11/2015].

, "Anuario Estadístico de Tamaulipas", México, 2014. En: http://www.datatur.sectur.gob.mx/ITxEF_Docs/TAMS_ANUARIO_PDF.pdf [Consultado 12 de octubre2015].

, "Anuario Estadístico y Geográfico por Entidad Federativa", México, 2014. En: http://internet.contenidos.inegi.org.mx/contenidos/productos/prod_serv/contenidos/espanol/bvinegi/productos/ anuario_14/702825066482.pdf [Consultado 17 de noviembbre 2015]. 
, "Directorio Estadístico Nacional de Unidades Económicas", México. En: http://www.beta.inegi.org.mx/app/mapa/denue/, [Consultado 16 de octubre 2015].

Jianu D. Muresan y Michail Vicentiu, "Controversies Regarding Cost, Uncertainties and Benefits Specific to Shale Gas Development", Sustainability, 2015, pp. 2473-2489.

Hui Jin and Stephen A. Sonnenberg (2014), "Characterization for Source-Rock Potential of the Bakken Shales in the Williston Basin", Colorado School of Mines, Article \#80356, North Dakota and Montana.

North Dakota State Water Commission, "Facts about North Dakota. Fracking and Water Use, 2016". En: http://www.swc.nd.gov/pdfs/ fracking_water_use.pdf, [Consultado 17 de septiembre 2016].

Pemex Exploración y Producción, "Aceite y Gas en Lutitas, Alcances de la Evaluación de su potencial en México", 2012.

PEMEX, "Aceite y Gas en Lutitas-Avances en la evaluación de su potencial en México", Pemex- PEP, 2012.

PROMÉXICO, "Tamaulipas-Mapa de Inversión en México", 2016. En: http://mim.promexico.gob.mx/swb/mim/Seleccion_de_indicadores, [Consultado 2 de octubre 2015].

Raitano Lucy, "Tampico-Misantla a basin that keeps on giving: IHS Markit”, Oil and Gas Mexico, México, 2017.

SEMARNART, "Guía de Criterios Ambientales para la Exploración y Extracción de Hidrocarburos Contenidos en Lutitas, 2015". En: http://biblioteca.semarnat.gob.mx/janium/Documentos/Ciga/ Libros2011/CD001945.pdf. [Consultado 22 de diciembre 2016].

USGS, Groundwater Science for a Changing World, "Water Used for Hydraulic Fracturing Varies Widely Across United States", Science for a changing world, 2015. En: http:/www.usgs.gov/newsroom/ article.asp?ID=4262\#.Voxbh_nhDIW, [Consultado 12 de diciembre 2016]. 
